\title{
Incidence and Mortality of Breast Cancer and their Relationship with the Human Development Index (HDI) in the World in 2012
}

\author{
Mahshid Ghoncheh ${ }^{1}$, Maryam Mirzaei ${ }^{2}$, Hamid Salehiniya ${ }^{3,4,5 *}$
}

\begin{abstract}
Background: Breast cancer is the most common malignancy in women worldwide and its incidence is generally increasing. In 2012, it was the second most common cancer in the world. It is necessary to obtain information on incidence and mortality for health planning. This study aimed to investigate the relationship between the human development index (HDI), and the incidence and mortality rates of breast cancer in the world in 2012. Materials and Methods: This ecologic study concerns incidence rate and standardized mortality rates of the cancer from GLOBOCAN in 2012, and HDI and its components extracted from the global bank site. Data were analyzed using correlation tests and regression with SPSS software (version 15). Results: Among the six regions of WHO, the highest breast cancer incidence rate (67.6) was observed in the PAHO, and the lowest incidence rate was 27.8 for SEARO. There was a direct, strong, and meaningful correlation between the standardized incidence rate and HDI $(r=0.725, p \leq 0.001)$. Pearson correlation test showed that there was a significant correlation between age-specific incidence rate (ASIR) and components of the HDI (life expectancy at birth, mean years of schooling, and GNP). On the other, a non-significant relationship was observed between ASIR and HDI overall ( $r=0.091$, $\mathrm{p}=\mathbf{0 . 2 4 1}$ ). In total, a significant relationship was not found between age-specific mortality rate (ASMR) and components of HDI. Conclusions: Significant positive correlations exist between ASIR and components of the HDI. Socioeconomic status is directly related to the stage of the cancer and patient's survival. With increasing the incidence rate of the cancer, mortality rate from the cancer does not necessariloy increase. This may be due to more early detection and treatment in developed that developing countries. It is necessary to increase awareness of risk factors and early detection in the latter.
\end{abstract}

Keywords: Incidence - mortality - breast cancer - development - world

Asian Pac J Cancer Prev, 16 (18), 8439-8443

\section{Introduction}

Cancer is uncontrolled growth of cells, and occurs in any part of the body (Keyghobadi et al., 2015; WHO, 2015a).It is one the most important health problems in the world (Youlden et al., 2014). In 2012, there were 14.1 million new cases of cancer in the world. It is predicted to increase to 22 million new cases per years until two next decades. Cancer is an obstacle to progress and well-being in the societies (Stewart and Wild, 2014). It affects everyone (old and young, poor and rich, and men and women), and associated with the great burden on the communities and countries. It is also the major cause of death in all communities, especially in developing countries (WHO, 2015a). Breast cancer includes 1.67 million new cases per years and $25 \%$ of all types of cancers, and is the second common cancer (Ferlay et al., 2015). Despite advances in cancer research, diagnosis and treatment of breast cancer is a challenge worldwide. The global burden of the disease is directly related to aging of population and behaviors associated with the cancer, so that the number of women suffering from the cancer continues to rise (Cecilio et al., 2015). The incidence rate of breast canceris different from 27 per 100,000 in African and East Asia to 96 per 100,000 in west Europe (WHO, 2015b). The cancer is the fifth cause of death, with 522,000 deaths in 2012 (Ferlay et al., 2015). Many risk factors, such as older age, a positive family history, late menopause, early menstruation, obesity after menopause, late pregnancy, and a history of hormone therapy, are related to the cancer (WHO, 2015b).

Studies have been shown that economic and social situation is directly related to the stage at diagnosis and survival of the patients (Clegg et al., 2009). It is also has been found that the incidence of the disease is higher in developed countries then developing countries due

${ }^{1}$ Department of Epidemiology and Biostatistics, School of Public Health, Hamadan University of Medical Sciences, Hamadan, ${ }^{2}$ School of Public Health, Tabriz University of Medical Sciences, Tabriz, ${ }^{3}$ Minimally Invasive Surgery Research Center, Iran University of Medical Sciences, ${ }^{4}$ Department of Epidemiology and Biostatistics, School of Public Health, Tehran University of Medical Sciences, ${ }^{5}$ Students'Scientific Research Center, Tehran University of Medical Sciences, Tehran, Iran *For correspondence: alesaleh70@ yahoo.com 
to increasing life expectancy, urbanization, and higher exposure to risk factors (WHO, 2015b). To determine progress and human development of the countries, the Human Development Index (HDI) is used by the United Nations Development Program (UNDP). It is a composite index of longevity, knowledge, and standard of living. Longevity is measured with life expectancy at birth, knowledge with potential years of education, and adequate standard of living with income or Gross National Product (GDP) per capita. HDI is a number between zero and one. According to HDI, countries in the world are divided into four categories, as follows: Countries with very high HDI (HDI $>=0.9)$, countries with a high HDI $(\mathrm{HDI}>=0.8)$, medium HDI countries $(0.8>\mathrm{HDI}>0.5)$, and countries with a low HDI (HDI $<=0.5)$ ([UNDP], 2014). This study aimed to investigate the relationship between HDI, and the incidence and mortality rates of breast cancer.

\section{Materials and Methods}

This study was an ecologic study in the world for assessment the correlation between age-specific incidence and mortality rate (ASR) with Human Development Index (HDI) and its details that include: Life expectancy at birth, Mean years of schooling and Gross national income (GNI) per capita. Data about the age-specific incidence and mortality rate (ASR) for every country for year 2012 get from global cancer project that available in (http://globocan.iarc.fr/Default.aspx) and Human Development Index (HDI) from Human Development Report 2013(Malik, 2013) that include information about HDI and its details for every country in the word for year 2012.

Method of estimate the age-specific Incidence and mortality rates in global cancer project by international agency for research on cancer

\section{Age-specific incidence rate estimate}

The methods of estimation are country specific and the quality of the estimation depends upon the quality and on the amount of the information available for each country. In theory, there are as many methods as countries, and because of the variety and the complexity of these methods, an overall quality score for the incidence and mortality estimates combined is almost impossible to establish. However an alphanumeric scoring system which independently describes the availability of incidence and mortality data has been established at the country level. The combined score is presented together with the estimates for each country with an aim of providing a broad indication of the robustness of the estimation.

The methods to estimate the sex- and age-specific incidence rates of cancer for a specific country fall into one of the following broad categories, in priority order:

1- Rates projected to 2012 (38 countries)-2- Most recent rates applied to 2012 population (20 countries)3-Estimated from national mortality by modelling, using incidence mortality ratios derived from recorded data in country-specific cancer registries (13 countries)-4Estimated from national mortality estimates by modelling, using incidence mortality ratios derived from recorded data in local cancer registries in neighboring countries (9 European countries)-5-Estimated from national mortality estimates using modelled survival (32 countries)-6Estimated as the weighted average of the local rates (16 countries)-7- One cancer registry covering part of a country is used as representative of the country profile (11 countries)-8-Age/sex specific rates for "all cancers" were partitioned using data on relative frequency of different cancers (by age and sex) (12 countries)-9- The rates are those of neighboring countries or registries in the same area (33 countries) (Jemal, 2011; Ferlay et al., 2015).

\section{Age-specific mortality rate estimate}

Depending of the degree of detail and accuracy of the national mortality data, six methods have been utilized in the following order of priority: 1-Rates projected to 2012 (69 countries)-2- Most recent rates applied to 2012 population (26 countries)-3- Estimated as the weighted average of regional rates ( 1 country)-4- Estimated from national incidence estimates by modelling, using country-specific survival (2 countries)-5-Estimated from national incidence estimates using modelled survival (83 countries)-6-The rates are those of neighboring countries or registries in the same area (3 countries) (Jemal, 2013).

\section{Human Development Index (HDI)}

Human Development Index (HDI), a composite measure of indicators along three dimensions: life expectancy, educational attainment and command over the resources needed for a decent living. All groups and regions have seen notable improvement in all HDI components, with faster progress in low and medium HDI countries. On this basis, the world is becoming less unequal. Nevertheless, national averages hide large variations in human experience. Wide disparities remain within countries of both the North and the South, and income inequality within and between many countries has been rising.(Malik, 2013)

Statistical analysis: In this study, we use of correlation bivariate method for assessment the correlation between age-specific incidence and mortality rate (ASR) with Human Development Index (HDI) and its details that include: Life expectancy at birth, Mean years of schooling and Gross national income (GNI) per capita. Statistical significance was assumed if $\mathrm{P}<0.05$. All reported $\mathrm{P}$-values are two-sided. Statistical analyses were performed using SPSS (Version 15.0, SPSS Inc).

\section{Results}

\section{The incidence rate}

It was estimated 1, 671,149new cases of breast cancer in the world in 2012. Of the cases, 882.9(per $100,000)$ were attributed to less developed countries, while 793.7(per 100,000) were attributed to developed countries. According to Globocan. it is the most common cancer in women world, with the standardized incidence rateof 43.1 per 100,000 . It also includes $25.1 \%$ of all cancers.

Among the six regions of WHO, the highest breast cancer incidence rate (67.6) was observed in the PAHO, 
Incidence and Mortality of Breast Cancer and their Relationship with the Human Development Index in the World in 2012

and the lowest incidence ratewas 27.8 for SEARO. Considering the regions in terms of HDI, the highest incidence rate was 78.2in the regions with very high development, and the lowest incidence rate was 26.5 in the regions with the medium human development. The highest incidence rate (111.9) was seen in Belgium, and the lowest rate (9) was related to Mongolia and Lesotho in the world. The five countries with the highest standardized incidence rate (per 100,000) were Belgium (111.9), Denmark (105), Bahamas (98), and Netherlands (96), respectively.

According to the division of the continents, the highest incidence rates were 91.6and91.1 for Northern America and Western Europe, respectively. However, the lowest incidence rates were26.8and27 for Middle Africa and Eastern Asia, respectively (Map1).

\section{The mortality rate}

It was estimated521, 907cases of deaths from breast cancer in the world in 2012. Breast cancer is the second cause of cancer death, with the standardized mortality rate (ASMR) of 12.9 (per 100,000), after lung cancer in the world. Breast cancer with the mortality rate of 12.9 is the first cause of death from cancer in women.

Among the six regions of WHO, the highest standardized mortality rate (18.6) was observed in the EMRO, and the lowestratewas 7 for the WPRO. Considering the regions in terms of HDI, the highest mortality rate was 17 in the regions with low development, and the lowest rate was9.8in the regions with the medium human development. According to the division of the continents, the highest rate was 17, and the lowest rate was6.9 for Africa and Eastern Asia, respectively (Map2).

The five countries with the highest standardized

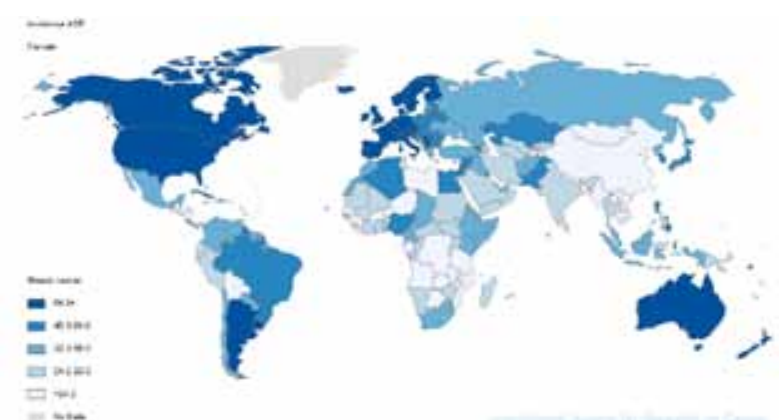

Map 1. Distribution of the Standardized Incidence Rate of Breast Cancer in World in 2012 (extracted from Globocan 2012)

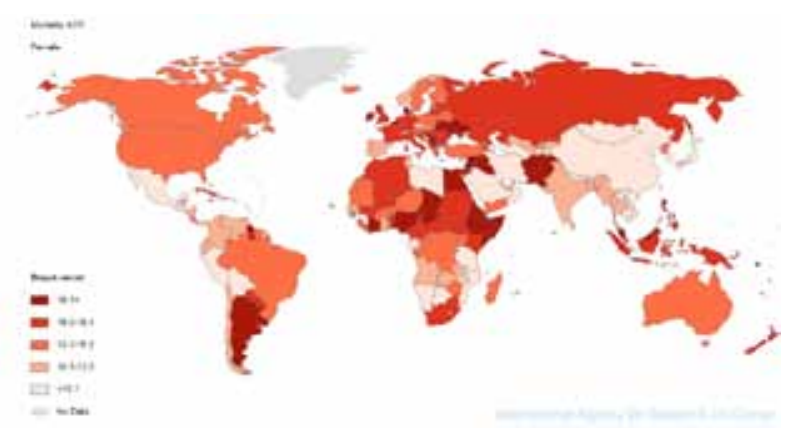

Map 2. Distribution of Standardized Breast Cancer Mortality Rates in World in 2012 (extracted from Globocan 2012) mortality rate (per 100,000)were Fiji (28.4), Bahamas (26.3), Nigeria (25.9), FYO Macedonia (25.6), and Pakistan(25.2), respectively (Map2).

\section{HDI}

Mortality to incidence ratio (MIR) was 0.31 for the cancer in the world. The ratio for the six regions of WHO was different from 0.21 in WPRO and PAHO, and 0.44 inEMRO to 0.65 in SEARO (Table 1). The highest and lowest MIRs were 0.56 and 0.16 for the continents such as the Middle Africa and Northern America.

As can see in Table 2, very high HDI countries had the highest ASR, equalto78, while low HDI countries had lowest ASR (32.6). Against, very high HDI countries had lower mortality rate (14.1) than low HDI countries (17). The lowest incidence and mortality was seen in countries with medium HDI.

Our findings also showed that there was a direct, strong, and meaningful correlation between the standardized incidence rate and HDI $(r=0.725, \mathrm{p} \leq 0.001)$ (Figure1). Pearson correlation test showed that there was a significant correlation between ASIR and components of the HDI (life expectancy at birth, mean years of schooling, and GNP) (Table2). On the other, a week and non-significant relationship was observed between ASMR and HDI

\section{Table 1. MIR in the Six Regions of W.H.O}

\begin{tabular}{llcl}
\hline Regions & ASR & AMSR & MIR \\
\hline AFRO & 34.5 & 17.2 & 0.5 \\
PAHO & 67.6 & 14 & 0.21 \\
EMRO & 41.9 & 18.6 & 0.44 \\
EUROP & 66.5 & 16 & 0.24 \\
SEARO & 27.8 & 12.9 & 0.46 \\
WPRO & 28.6 & 7 & 0.25 \\
\hline
\end{tabular}

Table 2. The Incidence and Mortality from Breast Cancer by HDI

\begin{tabular}{lccccc}
\hline Regions & \multicolumn{2}{c}{ Morbidity } & & \multicolumn{2}{c}{ Mortality } \\
\cline { 2 - 3 } \cline { 5 - 6 } & Numbers & ASR & & Numbers & MSR \\
\hline More developed & 788200 & 73.4 & & 197618 & 14.9 \\
Less developed & 882949 & 31.3 & & 324289 & 11.5 \\
Very High Human Development & 747203 & 78.2 & 169131 & 14.1 \\
High Human Development & 281687 & 45.2 & & 95646 & 14.6 \\
Medium Human Development & 493807 & 26.5 & & 182166 & 9.8 \\
Low Human Development & 147415 & 32.6 & & 74678 & 17 \\
World & 1671149 & 43.1 & 521907 & 12.9 \\
\hline
\end{tabular}

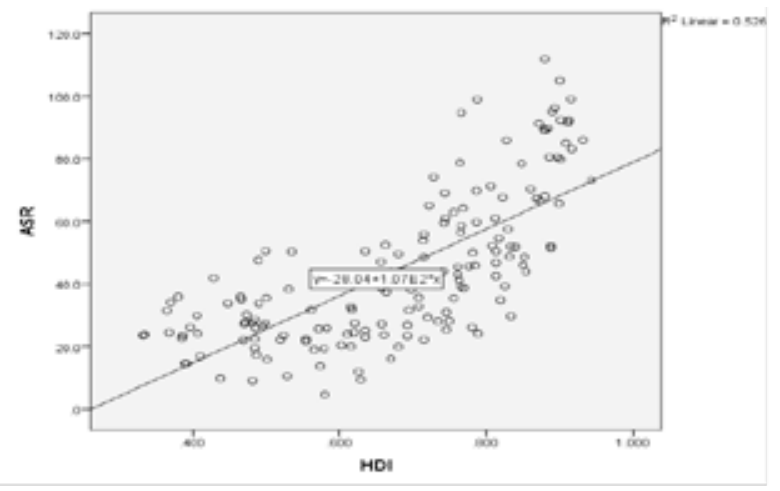

Figure 1. Correlation between HDI and Standardized Incidence of Breast Cancer in World in 2012 


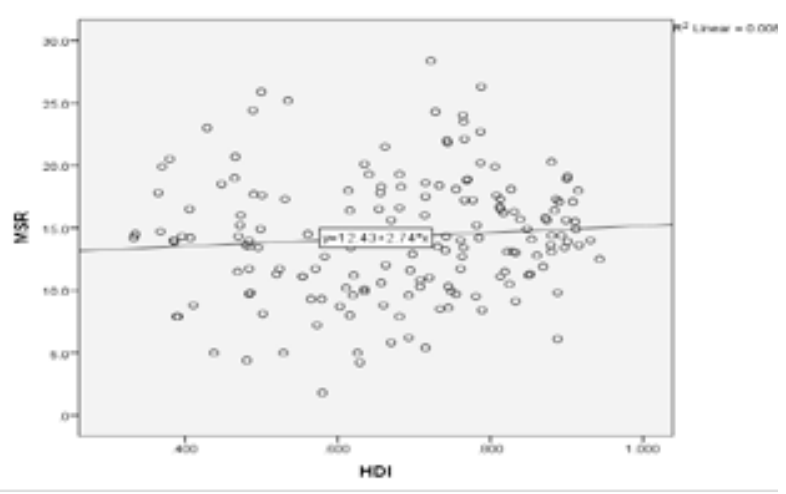

Figure 2. Correlation between HDI and Standardized Breast Cancer Mortality Rate in World in 2012

Table 3. Pearson Correlation between HDI Component and Dependent Variable

\begin{tabular}{lccccc}
\hline \multirow{2}{*}{ Variable } & \multicolumn{2}{c}{ ASIR* $^{*}$} & & \multicolumn{2}{c}{ ASMR $^{*}$} \\
\cline { 2 - 3 } \cline { 5 - 6 } life expectancy & $\mathrm{r}$ & $\mathrm{p}$-value & & $\mathrm{r}$ & $\mathrm{p}$-value \\
Mean schooling & 0.646 & $<0.001$ & & 0.049 & 0.527 \\
GNI & 0.404 & 0.005 & & 0.048 & 0.533 \\
& 0.575 & $<0.001$ & & 0.02 & 0.797 \\
\hline
\end{tabular}

*Dependent variables: ASIR and ASMR

$(\mathrm{r}=0.091, \mathrm{p}=0.241)$. In total, a significant relationship was not found between ASMR and components of HDI (Table3).

\section{Discussion}

Cancer is one of the most important health problems in the world (Stewart and Wild, 2014; Almasi et al., 2015; Razi et al., 2015). Breast cancer is very common in women in developed and developing countries (Clegg et al., 2009). Developed countries in West Europe face 96per 100,000for breast cancer. Although the incidence is lower in the less developed and developing countries, it will be increased, according to increasing life expectancy, increasing urbanization, and adoption of western lifestyle (WHO, 2015b). A study in India revealed that long-term living in the countryside and rural lifestyle reduces the risk of breast cancer (Nagrani et al., 2014). Prevention may somewhat reduce the risk of cancer, but this strategy cannot eliminate the majority of breast cancer in low and middle income countries, where breast cancer is diagnosed in very late stages. Therefore, early detection to improve the outcome of breast cancer treatment and survival is the cornerstone of breast cancer control. The5year survival of patients is very different in the world, so that it is more than 80 percent in North America and less than 40 percent in low-income countries (WHO, $2015 b)$. Socioeconomic status is directly correlated with disease stage and patient's survival (Clegg et al., 2009). Studies showed that patients with higher socioeconomic conditions are diagnosed earlier, and with controlling for other risk factors experience greater survival (Kish et al., 2014). Low survival of patients in developing countries is more related to the properties tumor, like the invasive tumor, and the lack of diagnostic and treatment facilities. As a result, the likelihood of developing more severe stages of the disease increases (Downing et al., 2007).
The researchers suggest that in low-income countries women should be considered in terms of education Breast Self-Examination (BSE). Although this method does not reduce deaths due to breast cancer, it increases women's awareness. Given the lack of resources in these countries, screening with mammography is questioned, and BSE can be continuously used (Albert and Schulz, 2003). Mammography in low and middle income countries is not more efficient and cost effective (Harford, 2011).

Unfortunately, in countries with limited resources, healthcare professionals are more in developed areas such as big and the central cities. Inadequate income, lack of appropriate job, lack of infrastructure, and working and living conditions lead to inappropriate distribution of specialists. To reduce this problem, governments must have sufficient effort so that all women can have access to healthcare (Kantelhardt et al., 2008).

According to the report of W.H.O, countries such as Fiji, Bahamas, Nigeria, and Pakistan with the highest death rates from breast cancer have no population-based national cancer registry (WHO, 2014).

Bray et al. also found the positive correlation between HDI and the cancer. In their study, it is shown that with increasing development, the incidence of cancers such as skin cancer increases, but the cancer associated with infectious agents reduces (Bray et al., 2012). In a study on HDI and breast cancer in Asia, it was also shown that the incidence of cancer increases with increasing HDI, and a significant positive correlation was observed between HDI and the incidence of the cancer, but this was not significant related to the death (Ghoncheh et al., 2014). Amit R. Patel et al. studied the relationship between HDI and kidney cancer, and found that the incidence and mortality of kidney cancer is reduced by increasing the HDI (Patel et al., 2012). One of the components of HDI is access to knowledge. A significant positive correlation between the knowledge and behavior of people in care and screening has been seen in studies (Chen et al., 2014). Mothers with low education receive less health care, and less likely to seek health care .Studies have shown that with increasing awareness, they more seek diagnostic and treatment services (Chen et al., 2014). Therefore, a positive relationship between cancer incidence and level of education could be due to early diagnosis.

In conclusion, The incidence of breast cancer is higher in countries with greater HDI than countries with lower HDI, while the mortality rate is greater in low HDI countries. There was no relationship between the incidence and mortality from the cancer. In other words, with increasing the incidence rate of the cancer, mortality rate from the cancer does not increase. Education of women is suggested in all countries for early detection and treatment.

\section{References}

[UNDP] UND. 2014. Human Development Report [Online]. UNDP. Available: http://www.undp.org/content/undp/ en/home/presscenter/events/2014/july/HDR2014.html [Accessed July,2014.

Albert US, Schulz KD (2003). Clinical breast examination: what can be recommended for its use to detect breast cancer in 
Incidence and Mortality of Breast Cancer and their Relationship with the Human Development Index in the World in 2012 countries with limited resources? Breast $J, 9,90-3$.

Almasi Z, Rafiemanesh H, Salehiniya H (2015). Epidemiology characteristics and trends of incidence and morphology of stomach cancer in Iran. Asian Pac J Cancer Prev, 16, 2757-61.

Bray F, Jemal A, Grey N, et al (2012). Global cancer transitions according to the Human Development Index (2008-2030): A population-based study. The Lancet Oncology, 13, 790-801.

Cecilio AP, Takakura ET, Jumes JJ, et al (2015). Breast cancer in Brazil: epidemiology and treatment challenges. Breast Cancer (Dove Med Press), 7, 43-9.

Chen GA, Tarver SL, Wen Y-F, et al (2014). Correlation Study of Knowledge and Behavior Regarding Breast Care among Female Undergraduate Students in China. Asian Pacific Journal of Cancer Prevention, 15, 10943-7.

Clegg LX, Reichman ME, Miller BA, et al (2009). Impact of socioeconomic status on cancer incidence and stage at diagnosis: selected findings from the surveillance, epidemiology, and end results: National Longitudinal Mortality Study. Cancer Causes Control, 20, 417-35.

Downing A, Prakash K, Gilthorpe M, et al (2007). Socioeconomic background in relation to stage at diagnosis, treatment and survival in women with breast cancer. British journal of cancer, 96, 836-40.

Ferlay J, Soerjomataram I, Dikshit R, et al (2015). Cancer incidence and mortality worldwide: sources, methods and major patterns in GLOBOCAN 2012. International Journal of Cancer, 136, 359-86.

Ghoncheh M, Mohammadian-Hafshejani A, Salehiniya H (2014). Incidence and mortality of breast cancer and their relationship to development in Asia. Asian Pacific journal of cancer prevention: APJCP, 16, 6081-7.

Harford JB (2011). Breast-cancer early detection in low-income and middle-income countries: do what you can versus one size fits all. Lancet Oncol, 12, 306-12.

Jemal A, Bray F, Center MM, et al (2011). Global cancer statistics. CA: A Cancer Journal for Clinicians, 61, 69-90.

Kantelhardt EJ, Hanson C, Albert U-S, et al (2008). Breast cancer in countries of limited resources. Breast Care, 3, 10-6.

Keyghobadi N, Rafiemanesh H, Mohammadian-Hafshejani A, et al (2015). Epidemiology and trend of cancers in the province of Kerman: southeast of Iran. Asian Pac J Cancer Prev, 16, 1409-13.

Kish JK, Yu M, Percy-Laurry A, et al (2014). Racial and ethnic disparities in cancer survival by neighborhood socioeconomic status in Surveillance, Epidemiology, and End Results (SEER) Registries. J Natl Cancer Inst Monogr, 2014, 236-43.

Malik K (2013). Human development report 2013. The rise of the South: Human progress in a diverse world. The Rise of the South: Human Progress in a Diverse World (March 15, 2013). UNDP-HDRO Human Development Reports.

Nagrani RT, Budukh A, Koyande S, et al (2014). Rural urban differences in breast cancer in India. Indian J Cancer, 51, 277-81.

Patel AR, Prasad SM, Shih Y-CT, et al (2012). The association of the human development index with global kidney cancer incidence and mortality. The Journal of urology, 187, 1978-83.

Razi S, Rafiemanesh H, Ghoncheh M, et al (2015). Changing trends of types of skin cancer in Iran. Asian Pac J Cancer Prev, 16, 4955-8.

WHO. 2014. World Health Organization - Noncommunicable Diseases (NCD) Country Profiles, 2014. [Online]. WHO. Available: http://www.who.int/nmh/countries/ fji_en.pdf?ua=1 2015].

WHO. 2015a. 10 facts about cancer [Online]. Available: http:// 